\title{
Polysèmes
}

Revue d'études intertextuelles et intermédiales

\section{The God of Small Things d'Arundhati Roy : entre saturation de couleurs et noir et blanc}

The God of Small Things by Arundhati Roy: from colour saturation to black and white

\section{Elsa Sacksick}

\section{(2) OpenEdition}

\section{Journals}

Édition électronique

URL : http://journals.openedition.org/polysemes/584

DOI : 10.4000 /polysemes. 584

ISSN : 2496-4212

Éditeur

SAIT

Référence électronique

Elsa Sacksick, "The God of Small Things d'Arundhati Roy : entre saturation de couleurs et noir et blanc », Polysèmes [En ligne], 14 | 2015, mis en ligne le 18 novembre 2015, consulté le 09 octobre 2020. URL : http://journals.openedition.org/polysemes/584 ; DOI : https://doi.org/10.4000/ polysemes.584

Ce document a été généré automatiquement le 9 octobre 2020.

Polysèmes 


\title{
The God of Small Things d'Arundhati Roy : entre saturation de couleurs et noir et blanc
}

\author{
The God of Small Things by Arundhati Roy: from colour saturation to black \\ and white
}

Elsa Sacksick

1 Bien que l'intrigue de The God of Small Things porte les couleurs sombres de la souffrance et de la perte de l'enfance, l'œuvre est pourtant profondément marquée par la couleur vive. Dès l'incipit, la description du paysage (l'Inde du Kerala) déborde de couleurs éclatantes reflétant la luxuriance de la nature, à tel point que certains critiques et détracteurs ont pu y voir une Inde "exotisée ", retravaillée pour faire "couleur locale ». En effet, la scène initiale donne à voir une palette de couleurs très large, du rouge au vert en passant par le noir, le jaune et le bleu des «bluebottles » : «black crows gorge on bright mangoes in still, dustgreen trees. Red bananas ripen. Jackfruits burst. Dissolute bluebottles hum vacuously in the fruity air [...] The countryside turns an immodest green. Boundaries blur as tapioca fences take root and bloom. Brick walls turn mossgreen " (GST 9) ${ }^{1}$. À l'image de l'incipit, tout le roman regorge de couleurs : «In their hooded yellow eyes there was a road and red flags marching. And a white shirt over a black back with a birthmark » (79), « Rahel's fingers were yellow green blue red yellow» (198). Le travail chromatique évoque presque le courant pictural du fauvisme avec ses taches de couleurs pures côte à côte qui ne cherchent pas à imiter le réel. Les scènes perçues à travers les yeux des deux jumeaux de sept ans en particulier sont teintées de couleurs irréelles, telle la scène à l'aéroport baignée de rouge ${ }^{2}$. La métaphore de la palette est d'ailleurs utilisée par le narrateur: «White-walled once. Red-roofed. But painted in weather-colors now. With brushes dipped in nature's palette. Mossgreen. Earthbrown. Crumbleblack » (306-307). Le travail des couleurs peut également évoquer les versions colorisées des films dont il est justement question dans l'intrigue (The Sound of Music notamment). The God of Small Things serait une sorte de roman en Technicolor. 
2 Ainsi, les couleurs éclatent, à l'instar de cet « immodest green » ou des fruits trop mûrs de l'incipit. Elles se juxtaposent sur le mode de la bigarrure, notion évoquant la multiplicité des couleurs, mais aussi leur mélange difficile, détonnant, voire dissonant. Or, la métaphore du mélange transgressif est centrale dans le roman. De même que la bigarrure évoque des couleurs qui ne peuvent se marier, de même, l'union impensable d'un intouchable avec une touchable est dissonante et l'union incestueuse des jumeaux est scandaleuse. Ainsi, les mélanges transgressent tantôt la morale, tantôt les codes sociaux des castes. La métaphore se décline encore avec le mélange culinaire, lui aussi frappé d'interdit, puisque les confitures confectionnées par l'usine familiale sont un mélange non autorisé auquel on refusera le label de confiture autant que de jelly: « neither jam nor jelly. Too thin for jelly, and too thick for jam » (30). Criardes, les couleurs le sont au sens pictural, mais aussi littéral. Souvent porteuses d'allitérations, elles acquièrent une qualité sonore, donnant à entendre la langue : "greygreen » (123, 293), « yellow rimmed red plastic sunglasses » ou « bluegreyblue eyes » (238). Dans les exemples qui suivent, la saturation sonore amplifie la saturation visuelle et ouvre même sur une saturation du sens : "white egg white» (104), « skyblue sky » (204) ou «finger-coloured fingers fought the ferns » (202). L'excès de couleur tend alors vers un épuisement du sens. L'ambivalence de la couleur et de son apport sémiotique apparaît déjà là. La question de la fonction de la couleur omniprésente, qui se situerait quelque part entre monstration et dissimulation, s'impose et va guider notre analyse.

3 Dans ce parcours chamarré, deux couples de couleurs, rouge/vert et noir/blanc, émergent et s'opposent quant à leur fonction et leur rapport au sens ou au dire. Dans un premier temps, le rouge et le vert rehaussent le roman et travaillent souvent de pair. Leur association, qui apparaît dès l'incipit: «brick walls turn mossgreen » ou "bright mangoes in dustgreen trees» (9), sera récurrente tout au long du roman: « red-veined, green [eyes]» (42), « red plastic shopping basket [...] green chillies » (11), « His voice was full of green rice fields and red banners » (121), " green the face, red the eyes » (233). Or, cette combinaison de couleurs qu'on appelle complémentaires est précisément associée aux jumeaux Rahel et Estha, personnages complémentaires s'il en est. En effet, tout au long du roman, le rouge correspond au personnage de Rahel: "[she was] seeing the world through yellow-rimmed red plastic sunglasses» (85). Certaines scènes, décrites de son point de vue, sont saturées de rouge, telle la manifestation communiste (pages 60 à 79) : « The air was red with flags. Rahel could see pieces of red sky. And in the red sky, hot red kites wheeled, looking for rats. In their hooded yellow eyes there was a road and red flags marching " (79) ou la scène dans l'usine: "Rahel put on her sunglasses. The factory was angry-coloured. The salted limes were red. The tender mangoes were red. The dusty sunbeam was red» (197) $»^{3}$. Cette couleur tache le roman d'une marque indélébile et sanguine, comme il tache les ongles vernis de Velutha juste avant son lynchage. La couleur rouge, tel un fil, traverse le roman et prépare ainsi le lecteur à la violence sanglante de la fin. Elle ménage un trajet pour l'œil du lecteur, comme le préconise Paul Klee à propos de la composition de ses tableaux. Les métaphores du narrateur ne cessent de renforcer cette symbolique du rouge : « The red had bled away » (13), « redly dead » (31), « blood red » (26).

4 Lorsque l'on regarde le monde à travers des lunettes rouges, il suffit de les retirer pour que tout apparaisse teinté de vert, sa couleur complémentaire ; on obtient alors le point de vue d'Estha, son jumeau. La scène à la gare où ce dernier est envoyé chez son père et séparé de sa mère et de sa sœur, est passée au filtre de la couleur verte : «a green-wavy 
[...] feeling " (323), « everything in the train was green. The seats green. The berths green. The floor green. The chains green. Darkgreen. Lightgreen. TO STOP TRAIN PULL CHAIN, it said in green. OT POTS NIART LLUP NIAHC, Estha thought in green » $(324)^{4}$. Le roman décline un véritable nuancier de verts : «dustgreen» (1), « wetgreen» (10), « slushgreen » (56), " greenwavy » (107), " greenheat » (172), « green-for-the-day » (187), « opaque green » (12), « greygreen » (123), « vomit green » (216). Dans ce roman à la focalisation instable, où le point de vue et l'énonciation glissent d'un jumeau à l'autre pour parfois inclure d'autres personnages, le rouge et le vert deviennent des couleurs repères, qui aident le lecteur à se retrouver dans les entremêlements de voix et les interruptions narratives.

5 En contrepoint de cette saturation de couleurs vives et visibles (et le fait qu'elles soient complémentaires les met plus encore en relief), un autre couple plus discret s'impose : le noir et blanc. Tandis que le rouge et le vert correspondent au couple gémellaire, le noir et le blanc - un duo antithétique - font écho au couple transgressif d'Ammu et Velutha (la «touchable » à la peau claire qui a une liaison avec l'intouchable à la peau noire). Le personnage de l'intouchable semble également synthétiser l'association noir et blanc: « He was called Velutha-which means White in Malayalam-because he was so black » (73) ou encore : « a white shirt over a black back » (79). Par ailleurs, alors que le rouge et le vert caractérisent plutôt l'univers diégétique et les descriptions visuelles, le noir et le blanc caractériseraient plutôt l'écriture. Opératoire du point de vue de la typographie, de la narration ou de la syntaxe, le «noir et blanc » serait une couleur stylistique qui signale le travail du vide dans le texte.

6 Dans un premier temps, l'association du noir et du vide apparaît à travers le leitmotiv des «black holes» (GST 82, 118, 191, 307), renforcé par la référence récurrente à « Heart of Darkness » $(54,328)$, aussi réappropriée par Roy en " dark of heartness » (306) : " A Joe-shaped hole in the Universe » (118), « He [Velutha] left behind a hole in the Universe through which darkness poured like liquid tar » (191), « a yawning, gaping hole. A History hole. A History-shaped hole in the Universe » (307), « God-shaped hole in the Universe $»^{5}$. Le motif est encore décliné avec l'image des taches qui représentent également la disparition et la mort:

Squashed Miss Mitten-shaped stains in the Universe.

Squashed frogs-shaped stains in the Universe.

Squashed crows that tried to eat the squashed frogs-shaped stains in the Universe

Squashed dogs that ate the squashed crow-shaped stains in the Universe (82).

Dans cet exemple la structure enfantine en imbrication et accumulation rend bien cette qualité expansionniste du trou noir qui absorbe et englobe tout alentour et qui fonctionne, pour les jumeaux, comme une représentation visuelle de l'absence et de la mort.

7 Si le trou noir incarne l'incompréhension des enfants face à la réalité violente des adultes, il représente en parallèle les zones d'ombre dans la compréhension du lecteur. Il devient alors une image cruciale pour rendre compte de la structure narrative. Le roman est en effet marqué par un trou de vingt-trois ans dans la trame temporelle, puisque le narrateur commence son récit en 1992 et se remémore les événements de 1969. Ce qui s'est passé entre ces deux périodes est à peine connu. Toute une série d'ellipses narratives émaillent le roman qui fonctionne à la manière d'un roman policier sur une information manquante. Certes, le lecteur apprend dès les premières pages que la jeune cousine anglaise (Sophie Mol) est morte et qu'il est arrivé quelque chose de tragique à Velutha; mais les circonstances exactes restent mystérieuses, 
opaques jusqu'aux trois quarts du roman. Ce noir, qui est aussi un «blank » dans le récit, représente en creux le meurtre de Velutha qui reste non dit jusqu'au chapitre 18.

Qui plus est, ce chapitre, véritable cœur du roman et des ténèbres qui recèle la scène traumatisante, fonctionne précisément comme cet espace astronomique dont la densité a le pouvoir d'attirer tout ce qui est autour. Il est, en effet, le point obscur vers lequel le récit converge et autour duquel il s'enroule, dans la mesure où la narration progresse en spirale autour de cette scène infernale qui laissera un vide dans le psychisme des enfants ${ }^{6}$.

9 La vacance dans la trame narrative fait écho à celle laissée dans le psychisme des jumeaux, témoins du meurtre. Ces derniers sont d'ailleurs décrits par une métonymie significative : "They sat there, Quietness and Emptiness ». Qu'il s'agisse du regard « the emptiness in her eyes " (54), ou de la parole: "a hollow where Estha's words had been " (19-20), ils sont tous deux hantés par le vide: «the emptiness in one twin was only a version of the quietness in the other " (20). Mais, le travail du vide dans le roman ne s'arrête pas là. Il se prolonge dans la langue et s'inscrit sur la page. C'est ici que le blanc rejoint le noir, il fonctionne comme l'autre face du même paradigme : le manque, le non-dit.

10 Les blancs ("blanks ») émaillent non seulement la trame narrative, mais également la trame syntaxique trouée par les ellipses de verbe. Les phrases nominales sont la marque de The God of Small Things et culminent dans le chapitre 18, frappé par un arrêt du temps (renforcé par la surabondance de phrases parataxiques) qui reflète l'état de sidération dans lequel se trouvent Rahel et $\mathrm{Estha}^{7}$, tous deux traumatisés par la violence du lynchage. Le paragraphe suivant témoigne de l'usage de la parataxe et de l'ellipse verbale :

The Kottayam Police. A cartoonplatoon. New-Age princes in funny pointed helmets.

Cardboard lined with cotton. Hairoil stained.

Their shabby khaki crowns.

Dark of Heart.

Deadlypurposed. (304)

Le roman semble perdre ses verbes pour accompagner la perte, littérale, du verbe chez Estha qui devient mutique.

11 Enfin, le blanc typographique peut se lire comme la représentation picturale de l'ellipse : l'inscription du vide à même la page. D'un point de vue formel, le lecteur ne peut qu'être frappé par sa présence visuelle. Roy joue avec la typographie, morcelle le texte en paragraphes, le blanc s'invite jusqu'à recouvrir une page entière, il noie le noir des mots, comme le montre l'exemple de la page 34 laissée blanche, avec une seule phrase flottant au milieu. La romancière structure son texte sur un mode visuel, elle organise les blancs de manière esthétique, donnant véritablement à voir l'écriture. En regardant la page, deux métaphores liées à la notion de vide peuvent venir à l'esprit : celle de la dentelle et celle de la peinture. En laissant volontairement des trous dans la page, l'auteur crée des "jours", terme qui désigne l'ouverture décorative produite dans le tissu en tirant les fils. Telle une dentellière, Roy joue avec les vides et les pleins. Les espaces blancs et les indentations graphiques tendent vers le calligramme. L'écriture se construit, dans ce sens, sur le même modèle que la parole de Rahel: « jagged like a piece of tin » (29):

Nothing.

On Rahel's heart Pappachi's moth snapped its sombre wings.

Out. 
In.

And lifted its legs.

Up.

Down. (293)

calligramme car, à force de répétition, le mot est vidé de son sens, et cet épuisement est rendu visible par son érosion typographique :
Nictitating
ictitating
itating
tating
ating
ting
ing. (189)

En parallèle, le blanc de la page évoque le procédé pictural de la réserve. La romancière s'applique à laisser des blancs, à la manière du peintre qui laisse des espaces non peints sur la toile. Or, le double sens du mot « réserve » en fait le maître mot du roman. Outre la partie blanche et vide de la toile, le terme évoque aussi la rétention, l'hésitation à dire, le non-dit. En ce sens, le silence s'infiltre dans le texte de manière visuelle. Meschonnic analyse le blanc comme le signe d'un silence latent: "La pleine page manifeste la prédominance du dire [...] ses modes sont innombrables. Mais tous s'opposent à un indicible qu'ils refoulent, éloignent. Quand le blanc vient, il note la limite transitoire du dit» (Meschonnic 304). Le blanc typographique dans The God of Small Things ne fait que révéler visuellement la stratégie narrative et syntaxique du non-dit. Tout le roman se construit sur la réserve, cette réticence à dire le traumatisme, en somme sur une esthétique du secret.

De manière significative, le texte est hanté par le silence qui acquiert une présence physique : «the silence sat between grand-niece and baby aunt like a third person. A stranger. Swollen. Noxious » (21), "the pickle smelling silence that lay between the twins like a bruise » (198) «Silence filled the car like a saturated sponge » (70), mais également une présence phonique grâce aux allitérations qui rendent le silence sonore : "silence dipped and soared and swooped and looped » (202), "a rushing, rolling, fishswimming silence " (293). On pourrait alors dire avec Meschonnic que The God of Small Things est « écrit d'une voix blanche » (Meschonnic 296).

Qui plus est, la réserve picturale attire aussi notre attention sur la fonction du blanc et du silence. En effet, si pour le peintre il agit comme une couleur en faisant ressortir les couleurs qui l'entoure, le blanc de l'écriture dans The God of Small Things est lui aussi un révélateur, il est signifiant. Par son vide éclatant, il attire l'œil (Pastoureau rappelle l'étymologie commune de «blank » et de "black» : la brillance) et finit par alerter le lecteur sur cette lacune, au-delà, à rebours des mots. Tandis que le narrateur s'ingénie à retarder le dire, la page ne cesse de montrer, de révéler la présence d'un secret. Celuici se dit en creux, en négatif. Finalement le blanc devient une réserve de sens, un indice laissé par la romancière; il ouvre littéralement une brèche dans l'espace du texte, de laquelle émerge l'ineffable traumatisme.

Le blanc s'impose ainsi comme la couleur la plus éloquente dans le roman; il comporte une dimension signifiante et esthétique, car il révèle non seulement le secret, mais aussi les mots : il rehausse leur charge poétique. Cette couleur instaure, en effet, un rapport privilégié entre les mots, ou, selon les termes de Paul Claudel, une «intimité 
directe » : « et voici, de quelques mots, débarrassés du harnais de la syntaxe et rejoints à travers le blanc par leur seule simultanéité, une phrase faite de rapports " (Claudel 699). Dans The God of Small Things, rehaussés de blanc, les mots résonnent. Claudel insiste encore sur la valeur sonore du blanc: «Laissons à chaque mot [...] l'espace - le temps - nécessaire à sa pleine sonorité, à sa dilatation dans le blanc» (701). Christine Buci-Glücksmann fait une remarque similaire à propos des silences dans la musique baroque : "Cet art du vide silencieux, comme point d'écriture et de respiration sonore ou visuelle permet à chaque note de rebondir, produisant de l'agrément " (BuciGlücksmann 56).

L'utilisation de la couleur dans The God of Small Things va alors à rebours de sa signification habituelle. Tandis que le blanc, couleur silencieuse et signe d'une lacune, guide le lecteur, le trop-plein de couleurs éclatantes devient aveuglant, il masque le sens et désoriente. Cette méfiance à l'égard des couleurs est une notion exprimée par Barthes lorsqu'il explique à propos de la photographie : «[...] j'ai toujours l'impression que la couleur est un enduit apposé ultérieurement sur la vérité originelle du noir et blanc. La couleur est pour moi un postiche, un fard» (Barthes 128). Dans le roman, comme dans un tableau impressionniste vu de trop près, les touches de couleurs disparates égarent l'œil et étourdissent le lecteur; ce n'est qu'en s'éloignant que le motif apparait. Les créations linguistiques de Roy sur la couleur (les doigts couleurs de doigt, le ciel bleu-ciel) proches du bégaiement, renforcent l'idée que dans la saturation de couleurs le sens se perd. Les couleurs présentent, par ailleurs, une fonction similaire au niveau des personnages: elles interviennent pour les détourner du réel et le transfigurer lorsqu'il devient insupportable et insensé, elles déréalisent. Estha voit tout en vert dans le train lorsqu'il est arraché à sa famille, il est tétanisé par les dents jaunes de son agresseur, le «Lemondrink Orangedrink Man », au cinéma : "the yellow teeth were magnets. They saw, they smiled, they sang, they smelled, they moved. They mesmerized» (102). Les allitérations en [s] viennent renforcer l'aspect hypnotique du jaune et, en tant que consonnes sourdes, créent un chuchotement insistant sur l'esthétique du secret.

18 L'oscillation entre débordement de couleurs et austérité du noir et blanc souligne la tension constante entre voir et dire, dans un roman où le drame se voit dans les blancs du texte avant d'être dit. Cette tension est également cristallisée par les personnages. Ceux qui voient sont condamnés au silence (Estha, renvoyé du cinéma et agressé parce qu'il a chanté au lieu de regarder le film, devient muet après avoir vu le lynchage, Velutha et Ammu sont tués ou exilés pour s'être vus de trop près) ${ }^{8}$, et c'est celui qui ne voit pas (le personnage borgne de Vellya Paapen) qui va dire le secret scandaleux de la liaison entre l'intouchable et Ammu. Le voir acquiert alors dans le roman une dimension médusante.

Tout, dans The God of Small Things, fait appel à l'œil. L'utilisation excessive et complexe de la couleur place le roman sous le signe du visuel et se trouve renforcée par les multiples références à la photographie, au cinéma ${ }^{9}$, ou encore par la construction très picturale de certaines scènes où règne l'hypotypose. Il faudrait, à ce titre, étudier plus en détail les lignes architectoniques de l'incipit et du début du chapitre 18 (aux pages 304-305) qui jouent sur le déplacement du regard le long d'un axe vertical en passant du ciel à la terre, et du minuscule au gigantesque. Le motif de l'œil est omniprésent et donne lieu à toutes sortes de métaphores surprenantes: « Her eyes spread like butter behind her thick glasses» (20), "She regarded Baby Kochamma with a beady-eyed 
interest. Her eyes were blurred jewels » (144), « They looked across the river with old Boat eyes " (204) ou encore "with sea-secrets in his eyes " $(92,227)$ où l'homophonie (sea/see) redouble le pouvoir du visuel. L'œil offre même une représentation métonymique des jumeaux : « Four saucers stared back at her [...]. Frightened eyes and a fountain looked back at her " (316). Qui plus est, le visuel contamine tous les autres sens comme le révèlent les multiples exemples de synesthésies: "a slow green whisper » (204), « listening with his eyes » (19 et 95), «listened with his flag » (71), « colored breeze » (216), « she could hear the blue cross stitch afternoon» (201).

Le roman se place sous le signe d'une véritable «folie du voir $»^{10}$, en réponse à l'impuissance du dire, qui culmine lorsque le borgne (Vellya Paapen) donne son œil de verre à l'aveugle (Mammachi). Entre les personnages pétrifiés par la vue du lynchage : " dinner-plated eyes they watched, mesmerized by something they didn't understand " (308), le foisonnement de couleurs qui désoriente, étourdit ou attire l'œil de manière hypnotique, se dessine le motif du regard comme piège qui fait de The God of Small Things une sorte de texte-gorgone qui méduse le lecteur.

\section{BIBLIOGRAPHIE}

Barthes, Roland. La Chambre claire. Paris : Seuil, 1980.

Buci-Glücksmann, Christine. La Folie du voir : de l'esthétique baroque. Paris : Éditions Galilée, 1986.

Claudel, Paul. Cuvre poétique. Paris : Gallimard, 1957.

Meschonnic, Henri. Critique du rythme : anthropologie historique du langage. Paris : Verdier, 1982.

Pastoureau, Michel. Noir : histoire d'une couleur. Paris : Seuil, 2013.

Roy, Arundhati. The God of Small Things. London: Flamingo, 1997.

\section{NOTES}

1. The God of Small Things apparaîtra désormais sous l'abréviation GST.

2. Voir pages 138-139.

3. Voir encore la scène au cinéma : « rush up the red steps with the old red carpet. Red staircase with red spit stains in the red corners " (97) ou celle des kangourous de l'aéroport (139).

4. Si le rouge est la couleur de la colère, celle que porte Rahel qui refuse de se conformer à la discipline dans son école, le vert est une couleur apaisante qui correspondrait à la position effacée d'Estha.

5. Voir aussi « Black cat-shaped holes in the universe » (82), « The grey, elephant-shaped hole in the Universe » (235).

6. Le roman commence en 1992 et va retourner progressivement par flashbacks jusqu'au cœur du drame en 1969. Il contourne en cercles concentriques ce point noir en suivant une spirale narrative. 
7. «Blue-lipped and dinner-plate-eyed, they watched, mesmerized by something they sensed but didn't understand » (308).

8. De même Rahel lorsqu'elle voit Velutha dans une manifestation communiste est punie et sommée de se taire.

9. Voir les nombreuses descriptions de photos ou la place centrale du film The Sound of Music dans l'intrigue.

10. L'expression est empruntée au titre de l'ouvrage de C. Buci-Glücksmann.

\section{RÉSUMÉS}

The God of Small Things est un roman saturé de couleurs dès ses premières lignes. Dans ce parcours bigarré, nous analyserons plus particulièrement deux couples de couleurs signifiants qui confèrent une qualité éminemment visuelle au roman : rouge/vert (correspondant au couple gémellaire de Rahel et Estha) et noir/blanc (renvoyant au couple transgressif de l'intouchable et de la touchable). L'analyse révèlera comment le roman remet en question le pouvoir éloquent de la couleur en opposition au noir et blanc qui finit par en dire plus sur le drame caché et retenu au cœur du récit. Le noir et le blanc fonctionnent en effet comme des couleurs « stylistiques » qui signalent le travail du vide dans le texte. Ils sont opératoires du point de vue de la typographie saturée de blanc sur la page, mais aussi du point de vue de la narration et de la syntaxe ponctuées de trous noirs avec les ellipses temporelles et verbales. Ils donnent ainsi véritablement à voir l'écriture et installent stylistiquement une esthétique du secret. L'oscillation entre saturation de couleurs et austérité du noir et blanc révèle une tension permanente entre voir et dire, ou plutôt entre ce qui se voit et ce qui ne se dit pas.

The God of Small Things is a novel saturated in colours from the very first page. In this mottled colour text we will analyse two sets of colours which are particularly meaningful and which confer the writing a very visual quality: red and green on the one hand (corresponding to the twins Rahel and Estha), and black and white on the other hand (referring to the transgressive couple of the untouchable man and the "touchable" woman). The analysis will reveal how the eloquent power of colour is questioned by the particular use of black and white. Indeed black and white turn out to reveal more about the untold drama concealed at the heart of the narrative and to work as "stylistic" colours enhancing the presence of void within the text. They are operating on the level of typography (white spaces saturate the page), but also on the level of narrative and syntax pitted with black holes (time and verb ellipses). Black and white thus confer a visual dimension to the writing and convey an aesthetics of secrecy. The oscillation between the saturation of colour and the black and white austerity reveals a permanent tension in the novel between seeing and saying, or more precisely between what can be seen and what cannot be said. 
INDEX

Keywords : black and white, aesthetics of secrecy, black holes, white spaces, lace, stylistics of colour

Mots-clés : stylistique de la couleur, blanc, noir et blanc, esthétique du secret, dentelle, trous noirs

oeuvrecitee God of Small Things (The)

\section{AUTEURS}

\section{ELSA SACKSICK}

Elsa Sacksick est Maître de Conférences à l'université Paris VIII (Vincennes), rattachée à l'équipe d'accueil de Paris VIII, EA 1569 " Transferts critiques et dynamiques des savoirs ». Elle a soutenu une thèse à la Sorbonne Nouvelle intitulée « Eloge de l'excès : tissage et hybridation dans l'œuvre de S. Rushdie, A. Roy et J. Winterson ». Elle a depuis publié de nombreux articles sur ces auteurs. Elle travaille sur les notions d'hybridité, d'animalité et d'oralité dans la littérature contemporaine britannique et indienne. Elle a participé récemment au premier colloque international consacré à Rushdie à Lisbonne en novembre 2013 avec la communication : « Roy and Rushdie: Cries and Whispers ». 